\title{
Juvenile Ossifying Fibroma of the Maxilla: A Case Report
}

\author{
Mahaboob Shaik $\cdot$ T. Kishore Babu • \\ G. PrabhuSekhar $\cdot$ V. Venkata Ramana Murthy
}

Received: 23 August 2011/Accepted: 27 September 2011/Published online: 18 October 2011

(C) Association of Oral and Maxillofacial Surgeons of India 2011

\begin{abstract}
Ossifying fibroma is a benign neoplasm of the bone, usually involving the posterior tooth bearing area of the mandible, predominantly seen in females in 2 nd -4 th decade of life with 5:1 prediliction. Fibro-osseous lesions other than FD seem to arise from the periodontal membrane. These lesions are usually asymptomatic, well defined clinically and radiologically amenable for enucleation. Fibro-osseous lesions of the jaws, including Juvenile Ossifying Fibroma (JOF), pose diagnostic and therapeutic difficulties due to their clinical, radiological and histological variability. Ossifying fibromas which appear as fast growing mass between 5 and 15 years of age, radiologically well bordered, and consistent with ossifying fibroma histologically, are referred as juvenile (aggressive) ossifying fibroma. We report a case of JOF of left side of the maxilla in an 11 year old girl which is an uncommon site of occurrence.
\end{abstract}

Keywords Juvenile ossifying fibroma $\cdot$ Maxilla . Fibroosseous · Tumors · Resection

M. Shaik · G. PrabhuSekhar · V. Venkata Ramana Murthy Department of Oral and Maxillofacial Surgery, St. Joseph Dental College, Eluru, Andhra Pradesh, India

M. Shaik $(\bowtie)$

Care Hospitals, D. No. 60-9-10, Siddhartha Nagar, Vijayawada,

Andhra Pradesh 520010, India

e-mail:dr.shaik786@yahoo.co.in

T. Kishore Babu

Department of Oral and Maxillofacial Surgery, Meenaakshi

Ammal Dental College, Chennai, Tamilnadu, India

\section{Introduction}

Fibro-osseous lesions of the cranial and facial bones are usually benign and tend to grow slowly. Benign fibroosseous lesions have similar histopathological features with fibrous dysplasia, ossifying fibroma, and cemento-ossifying dysplasia [1]. Ossifying fibroma, a rare tumor entity, is a well demarcated benign fibro-osseous tumor with capsule composed of metaplastic bone, fibrous tissue and varying amounts of osteoid. The ossifying fibromas are subdivided into conventional and juvenile clinicopathologic subtypes [2].

Conventional ossifying fibromas are usually slow growing and generally seen in the third and forth decades of life. They are treated with simple curettage and the recurrence is rare it affects people of all ages, but in contrast to the form seen at adults, the juvenile form is clinically more aggressive and tends to be recurrent. According to the new edition of the classification of the World Health Organization, ossifying fibromas which appear as fast growing mass between 5 and 15 years of age, radiologically well bordered, and consistent with ossifying fibroma histologically, are referred as juvenile (aggressive) ossifying fibroma. Juvenile ossifying fibroma (JOF) appears at an early age and in $79 \%$ of the patients is diagnosed before the age of 15 . JOF originates from periodontal ligament and ranges $2 \%$ of oral tumours in children [3].

The JOF is located mainly ( $85 \%$ ) in facial bones, in some cases $(12 \%)$ in calvarium and very seldom $(3 \%)$ extracranially. Ninety percent of the lesions located in the face region, involve the sinuses, mainly the maxillary antra. The tumor is well circumscribed by a tiny sclerotic shell of bone. It appears locally aggressive with cortical disruption and involvement of many adjacent anatomical structures. This lesion has predominating soft tissue consistency with variable amounts of 
internal calcification and/or linear or irregular focal bone. It usually shows a low density mass due to cystic changes on computed tomography (CT) scans. Following intravenous injection of iodinated contrast, the lesion may show diffuse appearance enhancement. Histologically, JOF is characterized by the presence of cellular fibrous stroma, garland like bony strands and cementum particles. An accurate diagnosis of JOF is made by correlating the clinical, linear radiography, CT scan, and histopathological findings (Fig. 1) [4].

\section{Case Description and Results}

We present a clinical case of an 11 year young girl referred to the department of oral and maxillofacial surgery for evaluation of a mass in the left cheek complaining of swelling on the left side of the face since 4 years. The mass had first appeared 4 years ago as a pea-nut and was growing larger since 6 months. Her past medical history was normal. Physical examination revealed a hyperemic swelling about $4 \times 3 \mathrm{~cm}$ in size, causing facial asymmetry on the left side of face. Maxillary enlargement and an intraoral lesion which had almost obliterated the buccal sulcus with few primary teeth displacement (Fig. 2).

Assessment with palpation showed a hard, non tender mass with smooth surface adhered to the maxilla. The mouth opening of the patient was normal and there were no decayed teeth in the lesion area, but there was malocclusion associated with displaced primary teeth. There were no assessed pathological changes of the mucous membrane in the tumor region. Panoramic radiograph showed an irregular but well bordered, unilocular, expansive lesion of the left side of the maxilla with displaced deciduous molars. Imaging studies demonstrated a soft tissue mass in the superior left maxilla which invaded the left maxillary sinus. The incisional biopsy was taken from the lesion and the pathology process was reported as JOF. Diagnosis was based on the presence of trabeculae of fibrillar osteoid and woven bone fragments. Surgical excision was carried out conservatively and the entire lesion was removed in Toto as a single mass which is clearly defined and lobulated. Postoperative course was uneventful and the patient was followed up for 6 months without a history of recurrence (Fig. 3).

\section{Discussion}

Most benign fibro-osseous lesions of jaws are asymptomatic and slowly progressing. Moreover, an unusual clinical presentation with apparent aggressive and destructive growth may be expected when the lesion is encountered in

Fig. 1 Preoperative photos
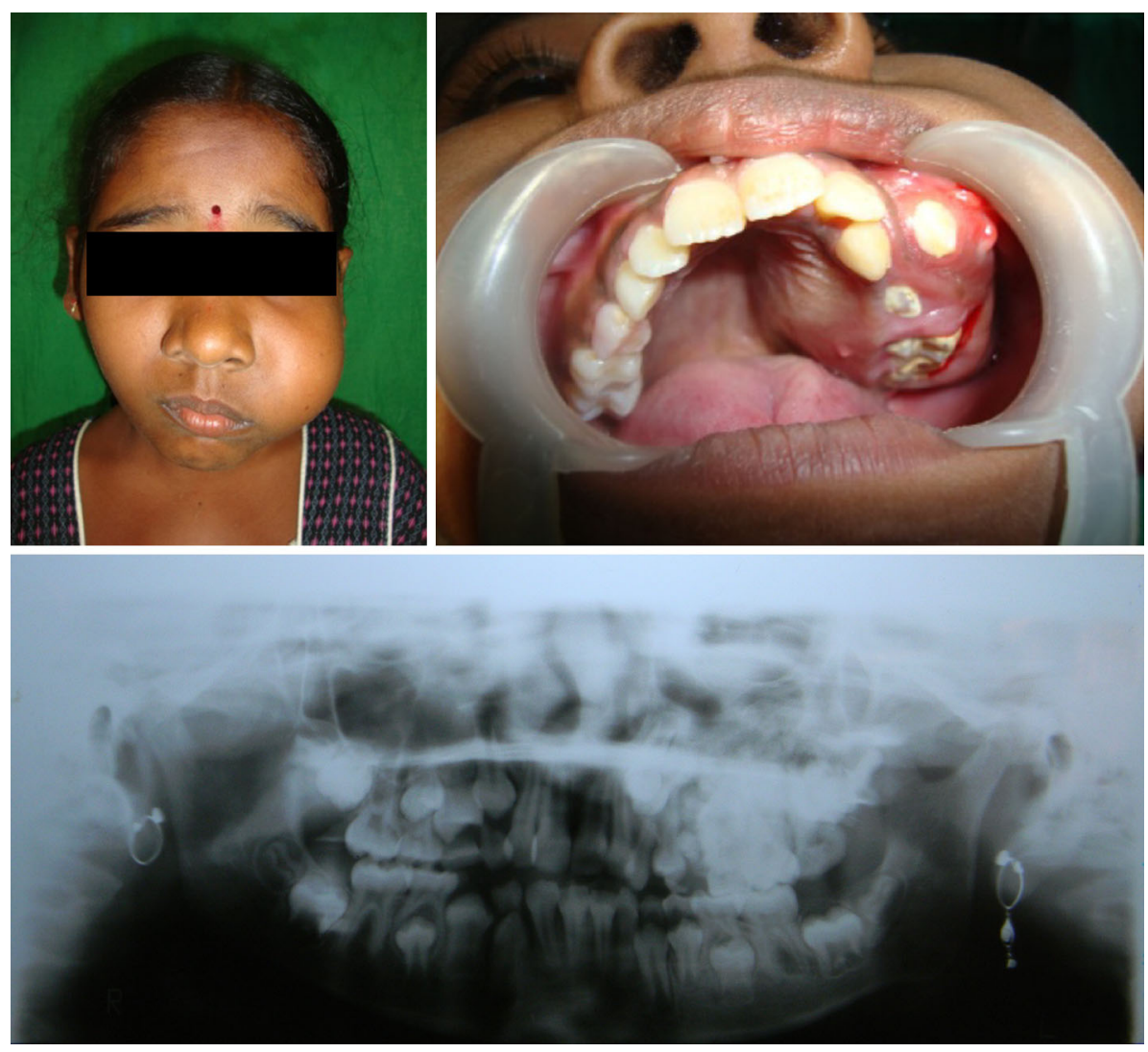
Fig. 2 Intra-operative photographs
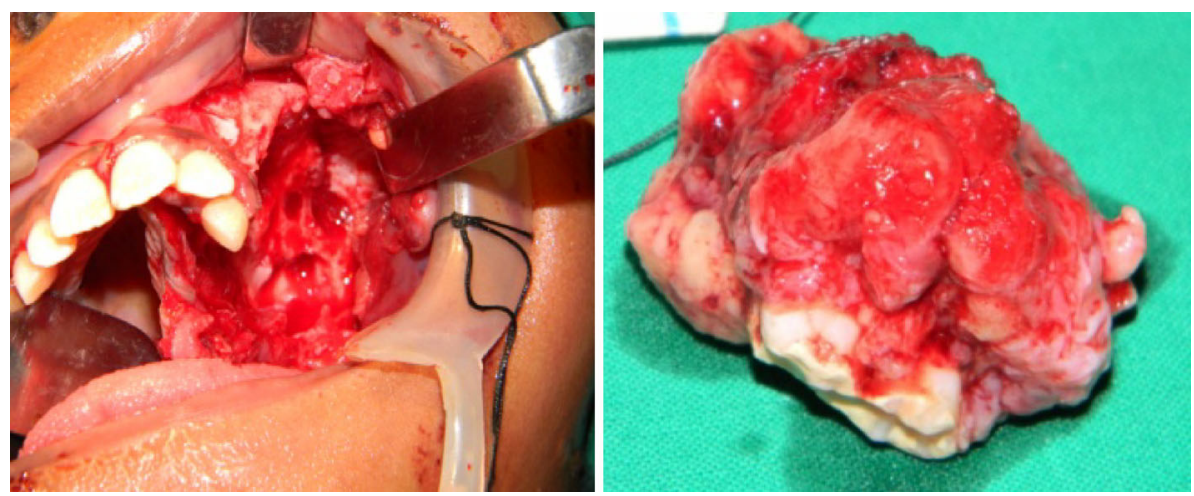
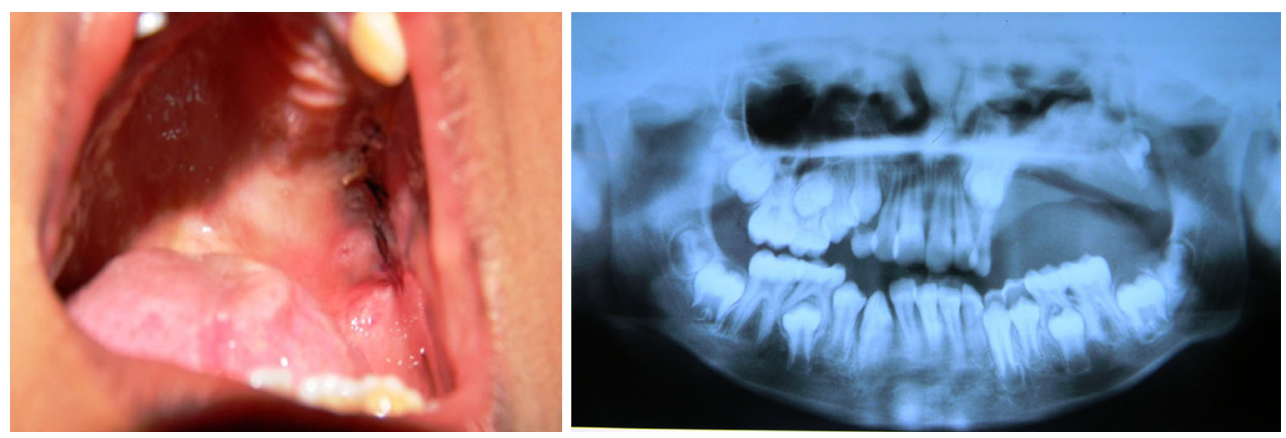

Fig. 3 Postoperative radiographs

a younger patient, especially below the age of 15 years [4]. The JOF is a fibro-osseous lesion that occurs in the facial bones also called aggressive ossifying fibroma due to its aggressiveness and the high tendency to recur, unlike other fibro-osseous lesions, such as cemento-ossifying fibroma, which may resemble radiographically. Due to its distinct histological features, JOF has been recognized as a separate histopathological entity among the fibro-osseous group of lesions. JOF is a relatively rare fibro-osseous lesion of the jaws characterized by the early age of onset i.e., under 15 years of age, the location of tumour, and the radiological appearance and the tendency to recur [5].

As the tumor initially expands in the bone, it remains encapsulated and therefore well demarcated radiologically. But when it reaches certain large size, it may lose its encapsulation and infiltrates beyond its margins for a few millimeters. A few cases of facial trauma have been suggested as a possible etiologic factor in the JOF development. Furthermore, osteoblastoma, osteosarcoma and odontogenic tumours should be considered in the differential diagnosis of JOF. While the osteoblastoma is radiologically seen as cystic bone lesion with sclerotic boundary, abnormal soft tissue mass and aggressive bone destruction is seen in the osteosarcoma, and cystic lesion connected to premolar or molar teeth is seen in odontogenic tumours. The JOF is characterized as expansive, having defined sclerotic borders, locally aggressive and destructive at cortex on CT scan.
Treatment of cemento-ossifying fibroma generally has been by conservative enucleation/curettage or radical surgery. Slootweg and Muller [3], however, reported that there was no difference in outcome between patients treated in a more limited way and those treated by major surgery [6]. Conservative surgery is therefore recommended even if the tumour is large with bowing and erosion of the inferior border of the mandible as shown. Radical treatment of the tumour such as an en bloc resection should only be considered if there are recurrences due to its aggressive nature. There are several advantages for treating large ossifying fibromas conservatively [7]. For example, there is minimal morbidity after surgery, good bone formation and consolidation, no loss of sensation as well as no bone graft required from a second surgical site. In long term follow-up cases where bowing or contours formed by these large lesions do not disappear completely with time, surgical intervention such as aesthetic recontouring of the bone may then be taken into consideration or recommended [8].

In the present case, conservative surgical approach was planned owing to child age, esthetic, functional and growth considerations. The tumor measured $4 \times 3 \mathrm{~cm}$ in diameter but did not lose its encapsulation and is lobulated. This property of tumor made surgical excision easier as a single mass. The post surgical wound was thoroughly inspected to confirm any involvement towards medial (nasal), roof (floor of the orbit) and posterior wall of the maxillary sinus. 
Postoperative follow up was made for 6 months with no complication.

\section{Conclusion}

Vast majority of ossifying fibromas are found in the posterior region of the mandible, although cases have been reported in the other craniofacial bones. These lesions are asymptomatic until they cause expansion of bone. Ossifying fibromas in young children tend to be aggressive, so conservative surgical procedures poses a risk of recurrence however, segmental resections are advocated for larger lesions, as per the requirement to limit the facial disfigurement and loss of function so, conservative surgical approach must be planned and performed.

Acknowledgments Special acknowledgments are due to Dr. P. Kishore Nayak, Dr. Sanjiv C. Nair

\section{References}

1. Waldron CA (1985) Fibro-osseous lesions of the jaws. J Oral Maxillofac Surg 43:249-262

2. Waldron CA (1993) Fibro-osseous lesions of the jaws. J Oral Maxillofac Surg 51:828-835

3. Slootweg PJ, Muller H (1990) Juvenile ossifying fibroma: report of four cases. J Cranio-Maxillofac Surg 18:125-129

4. Test D III, Schow C, Cohen D, Tilson H (1976) Juvenile ossifying fibroma. J Oral Surg 34:907-910

5. Marx RC, Stern D (2003) Text book of oral and maxillofacial pathology: a rationale for diagnosis and treatment. Quintessence Publishing Co, Inc, p 789

6. Vander Waall I (1991) Diseases of the jaws. Diagnosis and treatment. Munksgaard, Copenhagen, pp 225-229

7. Hall E, Naylor G, Mohr R, Warnock G (1987) Early aggressive cemento-ossifying fibroma: a diagnostic and treatment dilemma. Oral Surg Oral Med Oral Pathol 63:132-136

8. Zachariades N, Vairaktaris E, Papanicolaou S, Triantafyllou D, Papavassilliou D, Mezetis M (1984) Ossifying fibroma of the jaws. Review of the literature and report of 16 cases. Int J Oral Surg $13: 1-6$ 